Canadian University Music Review

Revue de musique des universités canadiennes

\title{
Le labyrinthe visité : Le cactus rieur et la demoiselle qui souffrait d'une soif insatiable de Denys Bouliane
}

\section{Jean-Michel Boulay}

Numéro 15, 1995

URI : https://id.erudit.org/iderudit/1014396ar

DOI : https://doi.org/10.7202/1014396ar

Aller au sommaire du numéro

\section{Éditeur(s)}

Canadian University Music Society / Société de musique des universités canadiennes

\section{ISSN}

0710-0353 (imprimé)

2291-2436 (numérique)

Découvrir la revue

Citer cet article

Boulay, J.-M. (1995). Le labyrinthe visité : Le cactus rieur et la demoiselle qui souffrait d'une soif insatiable de Denys Bouliane. Canadian University Music Review / Revue de musique des universités canadiennes, (15), 126-153.

https://doi.org/10.7202/1014396ar
Résumé de l'article

L’abandon, il y a maintenant environ 25 ans, des techniques sérielles d'organisation du matériau musical a rendu très difficile, pour les jeunes compositeurs, le choix du type de langage à employer. Cette absence de lingua franca a poussé les musiciens à explorer quantité de façons nouvelles d'organiser leur musique. Plusieurs d'entre elles n'ont pas eu la vie longue, d'autres se sont avérées plus prometteuses. Nous nous penchons ici sur une solution intéressante au problème du contrôle harmonique proposée par un compositeur québécois, Denys Bouliane, et sur son utilisation dans une œuvre importante pour orchestre, Le cactus rieur et la demoiselle qui souffrait d'une soif insatiable.
All Rights Reserved @ Canadian University Music Society / Société de musique des universités canadiennes, 1995
Ce document est protégé par la loi sur le droit d'auteur. L'utilisation des services d'Érudit (y compris la reproduction) est assujettie à sa politique d'utilisation que vous pouvez consulter en ligne.

https://apropos.erudit.org/fr/usagers/politique-dutilisation/ 


\section{LE LABYRINTHE VISITÉ : LE CACTUS RIEUR ET LA DEMOISELLE QUI SOUFFRAIT D'UNE SOIF INSATIABLE DE DENYS BOULIANE}

\section{Jean-Michel Boulay}

L'abandon, il y a maintenant environ 25 ans, des techniques sérielles d'organisation du matériau musical a rendu très difficile, pour les jeunes compositeurs, le choix du type de langage à employer. Cette absence de lingua franca a poussé les musiciens à explorer quantité de façons nouvelles d'organiser leur musique. Plusieurs d'entre elles n'ont pas eu la vie longue, d'autres se sont avérées plus prometteuses. Nous nous pencherons ici sur une solution intéressante au problème du contrôle harmonique proposée par un compositeur québécois, Denys Bouliane, et sur son utilisation dans une œuvre importante pour orchestre, Le cactus rieur et la demoiselle qui souffrait d' une soif insatiable ${ }^{1}$.

Denys Bouliane est né à Grand-Mère en 1955, mais c'est à Québec qu'il a passé son adolescence et ses années d'université. Son premier apprentissage musical s'est fait en autodidacte, comme guitariste rock. Ce n'est qu'après ses études secondaires qu'il commence à étudier la musique de façon organisée. Bientôt, à l'École de musique de l'Université Laval, il s'initie à l'analyse, à l'écriture, au piano et à la composition. En 1981, il quitte le Québec pour l'Allemagne et, de 1981 à 1985, il est élève de György Ligeti à la Hochshule für Musik de Hambourg. Depuis, il vit à Cologne, où il consacre tout son temps à la composition.

C'est à partir de son arrivée en Allemagne que Bouliane commence à réfléchir sérieusement à l'organisation des hauteurs. Cette réflexion est provoquée par une insatisfaction profonde face au langage sériel postdarmstadtien, qui n'arrive pas à établir, ou qui parfois refuse tout simplement une communication viable avec l'auditeur. Mais Bouliane est également insatisfait des facilités minimalistes et néo-romantiques.

Bien que la rupture avec le langage hermétique des compositeurs européens des années 60 ne soit pas difficile à faire, les influences conservatrices sont plus difficiles à éviter. Bouliane, à l'époque de son départ pour l'Allemagne, prend

1 Une première version de certaines sections de cet article a été présentée au congrès annuel de la Société de musique des universités canadiennes à Kingston en 1991. L'auteur aimerait remercier Denys Bouliane pour l'envoi de certaines esquisses et pour ses nombreuses explications. 
conscience, comme plusieurs jeunes compositeurs d'aujourd'hui, de son attachement (et de celui du public, bien entendu) pour la direction et la fonctionnalité tonales. Mais il s'avère que cette relation avec la tonalité est fondamentalement différente de celle des compositeurs allemands qui l'entourent, qui, eux, doivent porter le lourd boulet de la grande tradition germanique. Pour Bouliane, la tonalité est tout simplement un moyen qui pourra peut-être lui permettre d'arriver à ses fins, soit de créer des illusions sonores, une musique en constante transformation sans qu'elle paraisse vraiment changer, somme toute une musique du paradoxe.

Bouliane décide alors de se servir du matériau tonal comme d'un point de départ structurel abstrait, qu'il pourra transformer en quelque chose de neuf, un peu comme le ferait un illusionniste. Il n'y a donc pas de place ici pour le néoromantisme ou pour la nouvelle simplicité, qui sont des retours à des formes d'expression du passé, assaisonnées au goût du jour. De plus, Bouliane refuse absolument de se laisser entraîner sur le chemin des musiques répétitives et autres minimalismes à la mode. Pas de bricolage, pas de visite, comme le dirait Pierre Boulez, chez l'antiquaire'2.

Bouliane concentre son attention sur les échelles diatoniques. Pourquoi ce travail sur les échelles ? Probablement parce qu'il réalise rapidement qu'on ne peut développer de système élaboré de transformation de la syntaxe tonale sans bientôt annihiler le sentiment de direction. Or, c'est là l'élément qu'il veut absolument conserver. Après une étude de la structure des échelles diatoniques, il développe une série de transformations qui lui permettront de changer graduellement chacune de ces échelles sans trop perdre de leur « couleur ». On remarquera encore une fois un souci de transformation continuelle, un amour pour les sens multiples, les jeux de mots musicaux.

Bouliane a utilisé son système de transformations modales dans de nombreuses œuvres à partir de ses Jeux de société (1981). Après une exposition de ce système, nous nous pencherons sur une œuvre récente, écrite en 1986 pour l'Orchestre symphonique de Québec, Le cactus rieur et la demoiselle qui souffrait d' une soif insatiable.

\section{Système des transformations modales}

Dans un article publié en mars 1988 dans la revue musicale québécoise Sonances, Peter Niklas Wilson, rédacteur en chef de la Neue Zeitschrift für Musik, montre, sans toutefois en expliquer le fonctionnement, l'application du

2 Conférence publique prononcée au Département de musique de l'Université d'Ottawa en mai 1991. 


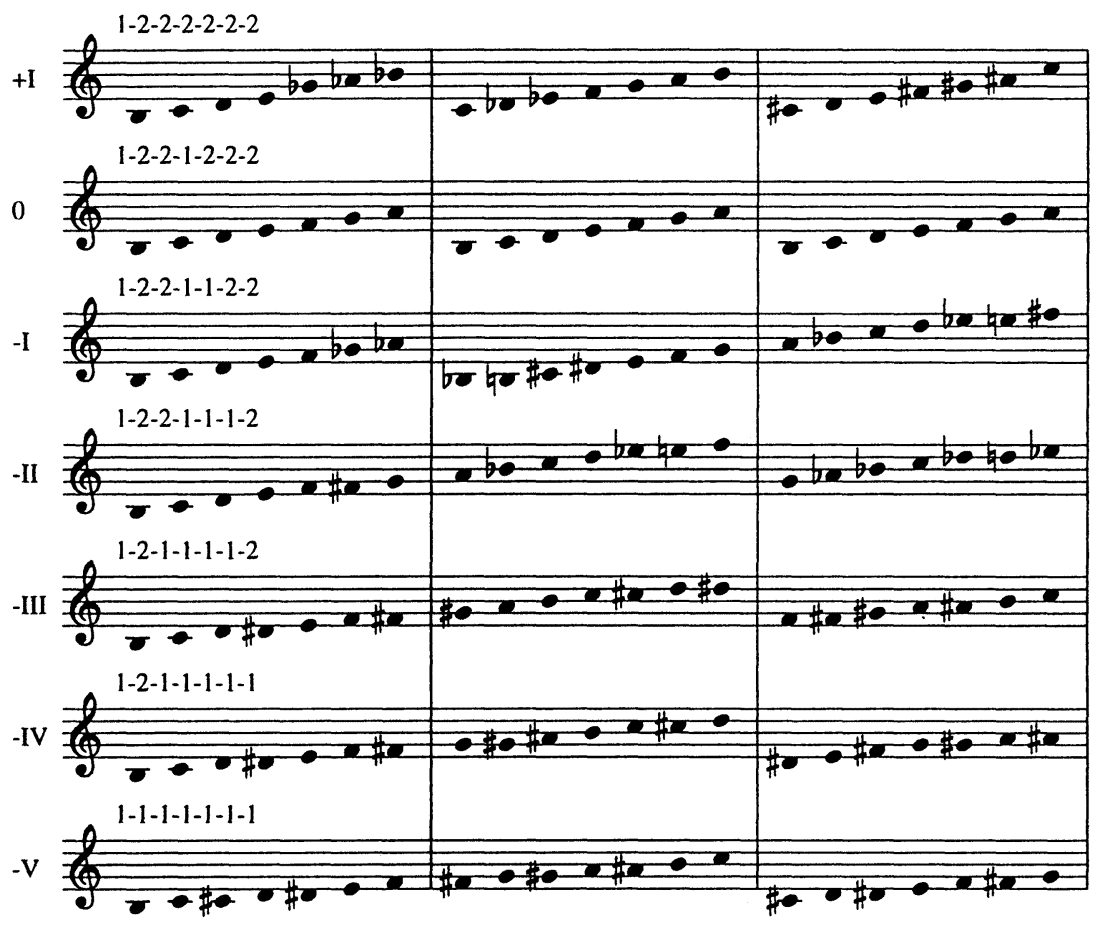

Exemple 1 : Transformations, mode de si.

transformations modales évoqué plus haut à l'échelle diatonique sur $d o^{3}$. L'exemple 1 montre les trois premières étapes des mêmes transformations, appliquées cette fois à l'échelle diatonique sur si.

La transformation 0 correspond à l'échelle surs $i$ dans sa forme originale, donc avec la succession d'intervalles 1-2-2-1-2-2-(2). Chaque groupe de sept notes (ensemble type 7-35) constitue un cycle complet ${ }^{4}$. Les transformations -I à -V, quant à elles, chromatisent graduellement l'échelle diatonique. En transformation -I, on remplace un intervalle de deux demi-tons par un intervalle de un demi-

3 Peter Niklas Wilson, « La musique du réalisme magique : portrait du compositeur Denys Bouliane ", Sonances 7, $\mathrm{n}^{\circ} 2$ (1988) : 22-33.

4 Nous employons ici la classification des ensembles de notes proposée par Allen Forte dans The Structure of Atonal Music (New Haven: Yale University Press, 1973). Dans la notation numérique employée, les chiffres 10 et 11 sont remplacés par les lettres $A$ et $B$. 
ton et la succession d'intervalles devient 1-2-2-1-1-2-(2). L'utilisation d'un intervalle de 2 pour revenir au début du groupe de sept notes crée un processus non octaviant qui devra être maintes fois répété avant que l'on puisse retomber sur ses pas, c'est-à-dire avant que l'on retrouve une échelle qui commence sur si. Ici, le cycle complet comprendra 12 étapes, puisqu'on a une transposition à $\mathrm{T}_{11}$ entre chaque ensembles de sept éléments (voir le tableau 1). La première note de chaque groupe de sept fera donc partie du cycle $\mathrm{C} 1$, dans la notation de Perle 5 . Chacun de ces groupes formera l'ensemble type 7-28. D'une étape du cycle à l'autre, on aura donc trois notes communes, formant l'ensemble type 3-5 [016] (voir le tableau 1 pour le processus complet).

En transformation -II, la succession d'intervalles devient 1-2-2-1-1-1-(2). L'ensemble de sept éléments est de type 7-14 et le cycle $\left(\mathrm{C}_{11}\right)$ comprend maintenant six étapes à cause des transpositions cycliques à $\mathrm{T}_{10}$. Les étapes adjacentes auront quatre notes communes, formant l'ensemble type 4-11 [0135]. La transformation -III est basée sur la succession d'intervalles 1-2-1-1-1-1(2), qui crée l'ensemble type 7-4 et un cycle de quatre étapes $\left(C 3_{11}\right.$, transposition à $T_{9}$ ). On retrouvera encore quatre notes communes entre étapes adjacentes, formant cette fois l'ensemble type 4-3 [0134].

En -IV, la succession d'intervalles 1-2-1-1-1-1-(1) crée le même ensemble de sept notes que celle de la transformation -III. Par contre, l'intervalle de jonction est maintenant un simple demi-ton (alors qu'il était un ton entier en-III). Le cycle a seulement trois étapes $\left(\mathrm{C}_{11}\right)$ puisque l'opération de transposition entre groupes est $\mathrm{T}_{8}$. Les étapes adjacentes ont en commun l'ensemble 3-2 [013]. Finalement, la transformation - $\mathrm{V}$ donne comme résultat la gamme chromatique elle-même. On retourne à un cycle de 12 étapes (comme en -I). À cause de l'uniformité totale des intervalles de la gamme chromatique, Bouliane n'utilisera pas cette transformation dans sa musique. La transformation $+I$, enfin, remplace le deuxième demi-ton de l'échelle par un ton entier, pour donner la succession 1-2-2-2-2-2-(2), soit une quasi-gamme par tons. L'ensemble type résultant est 7-33 qui, à $\mathrm{T}_{1}$ (cycle en 12 étapes), génère deux notes communes à distance de demi-ton.

Le compositeur a donc maintenant à sa disposition un moyen de transformer petit à petit des progressions tonales traditionnelles en progressions qui, bien que fondamentalement atonales, garderont un caractère pseudo-fonctionnel. Il peut aussi voyager de manière progressive entre deux divisions symétriques de l'octave : d'une part, une division en six parties, c'est-à-dire la gamme par tons, et d'autre part, une division en 12 parties, c'est-à-dire la gamme chromatique.

5 Voir George Perle,The Operas of Alban Berg (Berkeley : University of California Press, 1984), vol. 2, Lulu, 199. 
Tableau 1 : Transformations, mode de si (notation numérique)

\begin{tabular}{|c|c|c|c|c|c|c|c|c|c|c|c|c|}
\hline & $7-33$ & & & & & & & & & & & \\
\hline$+I$ & $\begin{array}{c}\text { B } 02468 \mathrm{~A} \\
\mathbf{7 . 3 5}\end{array}$ & 013579B & $12468 \mathrm{~A} 0$ & 23579B1 & $3468 \mathrm{~A} 02$ & $4579 \mathrm{~B} 13$ & $568 \mathrm{~A} 024$ & 679B 135 & $78 \mathrm{~A} 0246$ & $89 \mathrm{~B} 1357$ & $9 A 02468$ & A013579 \\
\hline 0 & $\begin{array}{c}\text { В024579 } \\
\mathbf{7 - 2 8}\end{array}$ & В024579 & В024579 & В024579 & B024579 & В024579 & В024579 & В024579 & B024579 & B024579 & B024579 & В 024579 \\
\hline$-I$ & $\begin{array}{c}\text { В } 024568 \\
7.14\end{array}$ & $\mathrm{AB} 13457$ & $9 A 02346$ & $89 \mathrm{~B} 1235$ & $78 \mathrm{~A} 0124$ & $679 B 013$ & $568 \mathrm{AB} 02$ & $4579 \mathrm{AB}$ & $34689 \mathrm{~A} 0$ & 235789B & $124678 \mathrm{~A}$ & 0135679 \\
\hline- II & $\begin{array}{c}\text { B024567 } \\
7-4\end{array}$ & $9 A 02345$ & $78 \mathrm{~A} 0123$ & $568 \mathrm{AB} 01$ & $34689 \mathrm{AB}$ & 1246789 & & & & & & \\
\hline -III & $\begin{array}{c}\text { B } 023456 \\
7.4\end{array}$ & $89 B 0123$ & 5689AB0 & 2356789 & & & & & . & & & \\
\hline$-I V$ & $\begin{array}{c}\text { B } 023456 \\
7-1\end{array}$ & $78 \mathrm{AB} 012$ & $346789 \mathrm{~A}$ & & & & & & & & & \\
\hline$-\mathrm{V}$ & B012345 & $6789 \mathrm{ABO}$ & 1234567 & $89 \mathrm{AB} 012$ & 3456789 & $\mathrm{AB} 01234$ & $56789 \mathrm{AB}$ & 0123456 & $789 \mathrm{AB} 01$ & 2345678 & $9 \mathrm{AB} 0123$ & $456789 \mathrm{~A}$ \\
\hline
\end{tabular}




\section{Modèle harmonique}

Pour voir comment Bouliane peut utiliser ce système de base à des fins de composition, prenons comme matériau générateur une progression typiquement tonale, soit un cycle de quintes diatoniques descendantes (toujours en mode de si), et dérivons-en les transformations. Dans l'exemple 2, on commence sur le dernier si et on extrait les quintes descendantes, en lisant donc de droite à gauche. Les éléments du cycle sont à quatre positions l'un de l'autre. Cette constatation nous permettra d'en extraire facilement les transformations. C'est ce qui est fait au tableau 2.

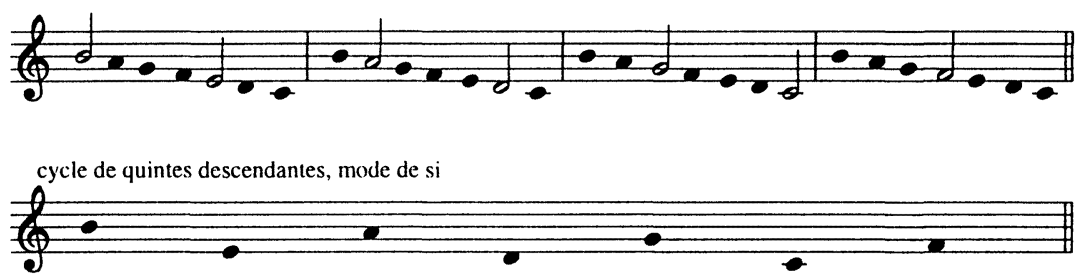

Exemple 2 : Extraction du cycle de quintes descendantes, mode de si.

Bien entendu, nous n'avons plus de véritables cycles de quintes mais bien des transformations plus ou moins chromatiques d'un modèle absolument diatonique. En transformation $+\mathrm{I}$, on retrouve un cycle d'intervalle $<4545455->$, ce qui donne une grande concentration de tricordes 3-11 [037]. En transformation -I, le cycle d'intervalles est $<6565666->$. Ici, on retrouve surtout le tricorde 3-5 [016]. En -II, le cycle d'intervalles $<7576676->$ crée aussi une forte concentration de tricordes de type 3-5. En -III, le cycle d'intervalles est $<7686777->$ et les tricordes sont maintenant de types 3-5, 3-8 [026] et 3-9 [027]. Finalement, en -IV, le cycle d'intervalles $<8787787->$ crée, comme en + I, un grand nombre de tricordes 3-11. Les transformations -IV et $+\mathrm{I}$ possèdent des cycles d'intervalles complémentaires (en mod 12) et peuvent donc être considérées comme les deux pôles du processus de transformation.

Nous pouvons maintenant prendre toute progression tonale basée sur ce cycle des quintes et en dériver les transformations en mode desi. Prenons par exemple la progression de dominantes altérées de l'exemple 3a, typique de la musique de jazz. Il s'agit d'une alternance des deux tétracordes contenant un exemplaire de chacun des intervalles, les deux tétracordes $Z$ de la classification de Forte, 4-15 [0146] et 4-29 [0137]. Ces deux tétracordes sont reliés par l'opération $T_{n} M$ (multiplication par 5), qui transforme une gamme chromatique en cycle de 
Tableau 2 : Extraction du cycle de quintes descendantes et de ses transformations, mode de si (lire de droite à gauche); transformation du cycle de quintes descendeantes, mode de $s i$.

\begin{tabular}{|c|c|c|c|c|c|c|c|c|c|c|c|c|c|}
\hline \multicolumn{14}{|c|}{ Extraction du cycle de quintes descendantes et de ses transformations, mode de si (lire de droite à gauche) } \\
\hline$+\mathbf{I}$ & B02468A & 013579B & $12468 \mathrm{~A} 0$ & 23579B 1 & 3468A02 & 4579B13 & $568 \mathrm{~A} 024$ & $679 B 135$ & $78 \mathrm{~A} 0246$ & $89 \mathrm{~B} 1357$ & $9 \mathrm{~A} 02468$ & A013579 & B \\
\hline $\mathbf{0}$ & B024579 & B024579 & B024579 & B024579 & B024579 & B024579 & B024579 & В024579 & В024579 & B024579 & В 024579 & B024579 & B \\
\hline$-I$ & В024568 & $\mathrm{AB} 13457$ & $9 \mathrm{~A} 02346$ & 89B1235 & $78 \mathrm{~A} 0124$ & $679 \mathrm{~B} 013$ & $568 \mathrm{AB} 02$ & $4579 \mathrm{AB} 1$ & 34689A0 & 235789 B & $124678 \mathrm{~A}$ & 0135679 & B \\
\hline- II & В024567 & $9 A 02345$ & $78 \mathbf{A} 0123$ & $568 \mathrm{AB} 01$ & $34689 \mathrm{AB}$ & 1246789 & B 024567 & $9 \mathrm{~A} 02345$ & $78 \mathrm{~A} 0123$ & $568 \mathrm{AB} 01$ & $34689 \mathrm{AB}$ & 1246789 & B \\
\hline -III & B023456 & $89 \mathrm{~B} 0123$ & $5689 \mathrm{ABO}$ & 2356789 & B023456 & $89 \mathrm{~B} 0123$ & $5689 \mathrm{ABO}$ & 2356789 & B023456 & $89 \mathrm{~B} 0123$ & $5689 \mathrm{ABO}$ & 2356789 & B \\
\hline$-I V$ & В023456 & $78 \mathrm{AB} 012$ & $346789 A$ & B023456 & $78 \mathrm{ABO} 12$ & $346789 A$ & В 023456 & $78 \mathrm{AB} 012$ & $346789 A$ & B023456 & $78 \mathrm{AB} 012$ & $346789 \mathrm{~A}$ & B \\
\hline
\end{tabular}

Transformations du cycle de quintes descendantes, mode de $s i$
+I B3805927B4815A3704916
0 B492705
-I B5A4939392817171605B5
-II B6B606172728293A3A4A5
-III B608294
-IV B72A5083B6294073A6184 
quintes $^{6}$. Chacune des trois voix supérieures de l'exemple 3a est un segment chromatique descendant. On peut aussi «diatoniser» cette progression en transformant chacun de ces segments chromatiques en segment descendant du mode de $s i$, comme à l'exemple $3 \mathrm{~b}$.

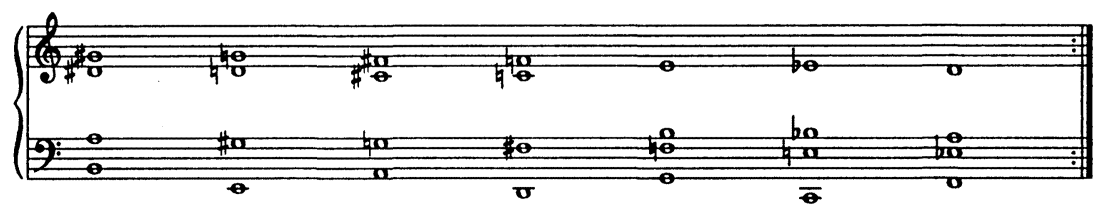

Exemple 3a : Progression modèle.

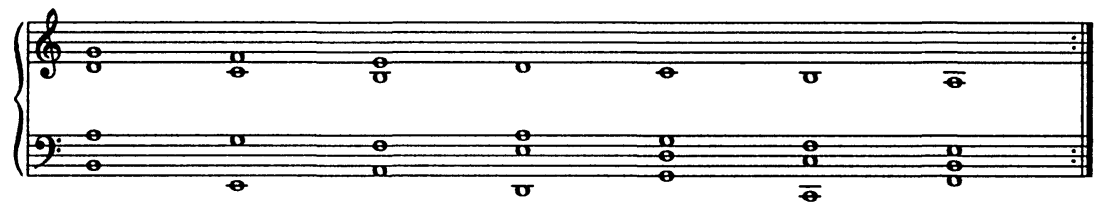

Exemple 3b : Progression diatonique, mode de si.

Il est maintenant possible de dériver facilement toutes les transformations, comme à l'exemple $4^{7}$. Puisque chaque voix de l'exemple $3 \mathrm{~b}$ est une descente diatonique en mode de $s i$, chacune des voix des progressions transformées consistera en une descente des échelles présentées à l'exemple 1. Puisque plusieurs de ces transformations ont des cycles particulièrement longs, l'exemple 4 ne donne, pour des raisons d'espace, que les 21 premiers accords de la progression transformée.

La prochaine étape sera de considérer chacune de ces sonorités comme un ensemble de notes pouvant être « recomposé » dans différents registres. Bouliane décide, de façon arbitraire, de ne pas utiliser la ligne de basse, la transformant du même coup en « pseudo-basse fondamentale ». Il réarrange ensuite les trois voix supérieures comme à l'exemple 5. Il possède donc maintenant cinq versions d'un modèle harmonique à trois parties. Chaque version complète du modèle contient 84 accords, ce qui nous donne une matrice harmonique à 420 positions $(84$ x 5).

6 Pour en savoir plus sur $\mathrm{T}_{\mathrm{n}} \mathrm{M}$ et $\mathrm{T}_{\mathrm{n}} \mathrm{MI}$, voir Robert Morris, Class Notes for Atonal Music Theory (Hanover : Frog Peak Music, 1991), 58-62.

7 Dans les exemples 4 et 5 , les altérations ne s'appliquent que pour la note devant laquelle elles sont placées. 
Progression de quintes descendantes, mode de si (Transformation +1 )

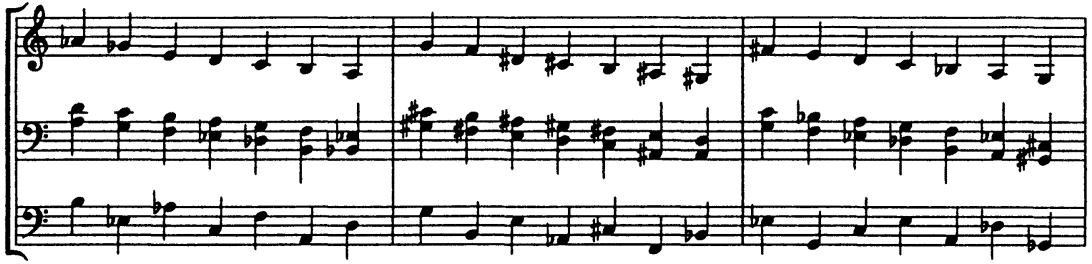

Progression de quintes descendantes, mode de si (Transformation -I)

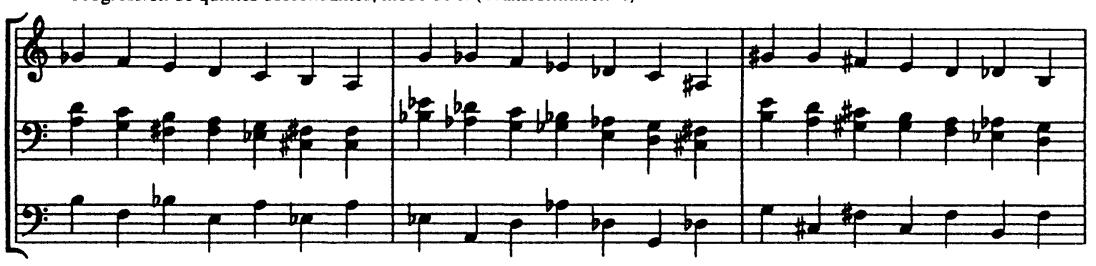

Progression de quintes descendantes, mode de si (Transformation -II)

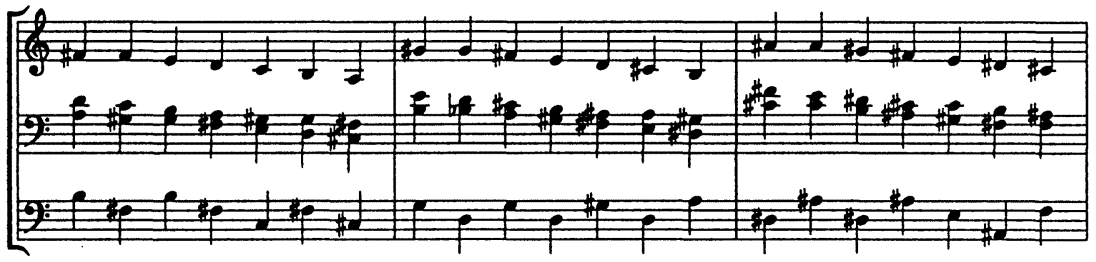

Progression de quintes descendantes, mode de si (Transformation -III)

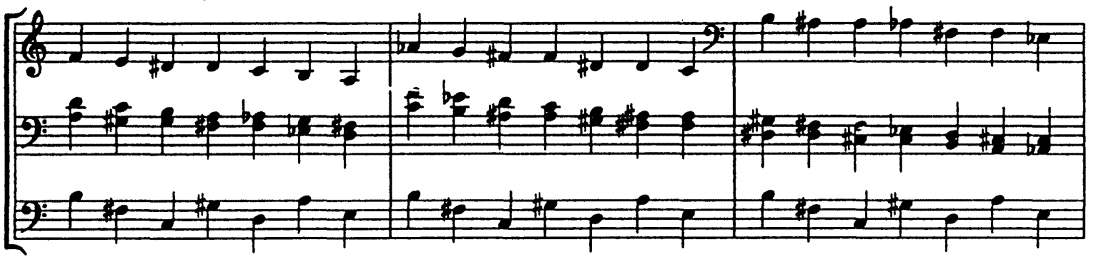

Progression de quintes descendantes, mode de si (Transformation -IV)

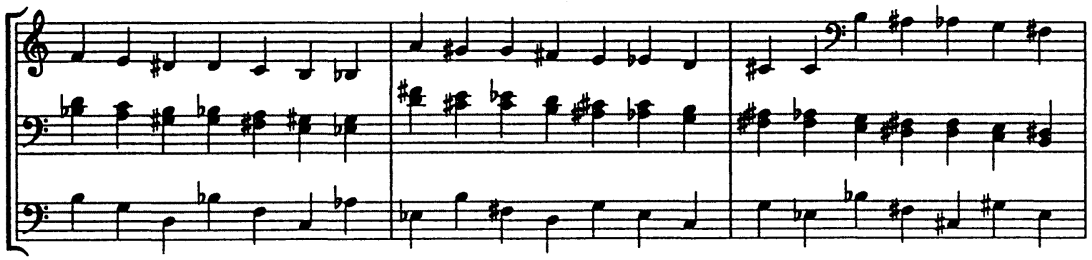

Exemple 4 : Transformations de la progression diatonique, mode de $s i$. 
Modele harmonique (Transformation +1 )

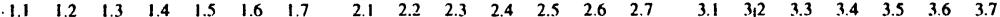

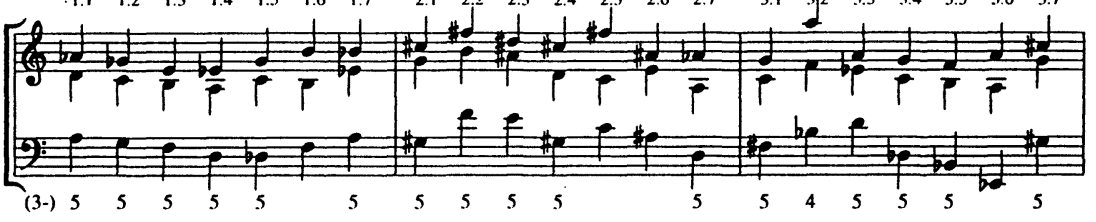

Modèle harmonique (Transformation -I)

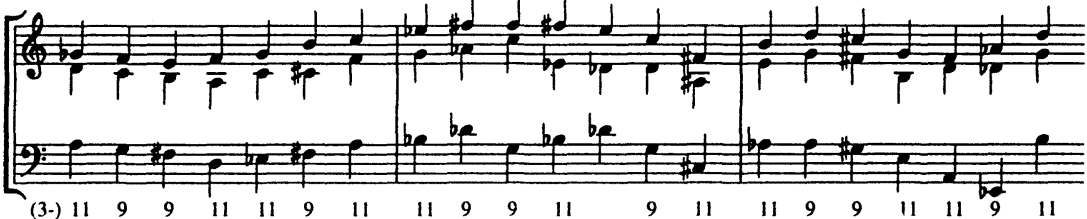

Modèle harmonique (Transfornation -11)

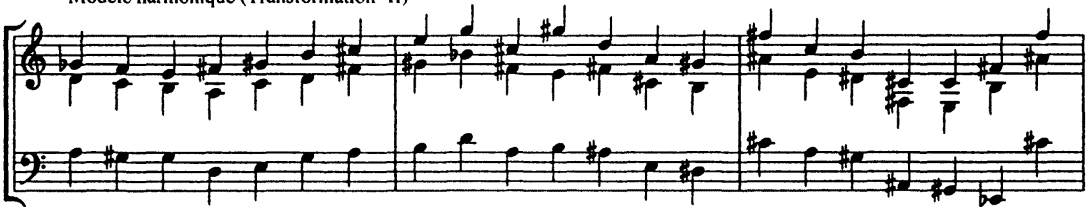

$\begin{array}{llllllllllllllllllllllllllll}(3-) & 11 & 11 & 11 & 11 & 12 & 11 & 11 & 11 & 11 & 11 & 11 & 12 & 11 & 11 & 11 & 11 & 11 & 11 & 12 & 11 & 11\end{array}$
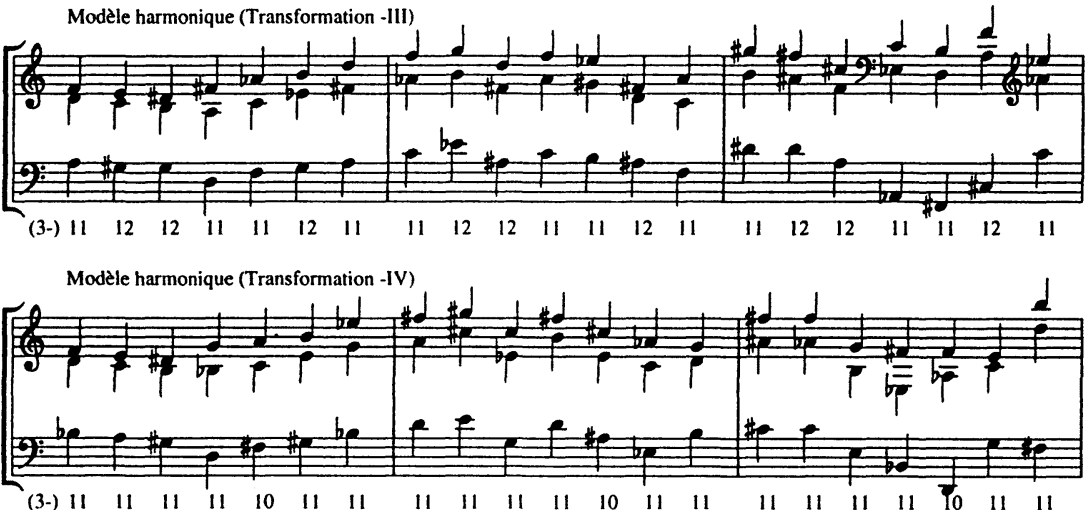

$\begin{array}{lllllllllllllllllllllllllll}(3-) & 11 & 11 & 11 & 11 & 10 & 11 & 11 & 11 & \| 1 & 11 & 11 & 10 & 11 & 11 & 11 & 11 & 11 & 11 & 10 & 11 & 11\end{array}$

Exemple 5 : Transformations du modèle harmonique, mode de si. 
Encore une fois, pour des raisons d'espace, l'exemple 5 ne donne que les 21 premiers accords de cette matrice ${ }^{8}$.

Examinons d'un peu plus près le matériau harmonique ainsi créé. En transformation $+I$, la grande majorité des accords de trois sons sont de type 3-5 [016], c'est-à-dire qu'ils contiennent un exemplaire des intervalles 1,5 et 6 . Le sixième accord de chaque groupe de sept ne contient que deux notes formant triton. En transformation -I, on obtient une combinaison d'accords parfaits (tricordes de type 3-11 [037]) et d'accords de type 3-9 [027], qui contient un intervalle 2 et deux intervalles 5 . En -II, on retrouve surtout des accords parfaits. Le cinquième accord de chaque groupe de sept est un accord augmenté (tricorde de type 3-12 [048]). En -III, l'accord augmenté prend un peu plus d'importance. Une comparaison avec la transformation -I montre que les accords parfaits sont distribués aux mêmes endroits et que les accords augmentés viennent remplacer les tricordes 3-9. Finalement, en -IV, l'accord augmenté de la transformation -II est remplacé par un accord diminué (tricorde de type 3-10 [036]).

On aura remarqué l'omniprésence des accords classés dans cette matrice harmonique. C'est grâce à cette grande concentration de sonorités traditionnelles dans un contexte absolument nouveau et original que Bouliane arrivera à créer une musique où l'auditeur ne se sentira pas totalement désorienté. Il pourra y avoir une augmentation du rythme harmonique puisque les accords resteront en somme assez simples.

Bouliane utilisera ce modèle harmonique de multiples façons. Il pourra, par exemple, en faire une lecture horizontale, donc conserver une homogénéité quant à la transformation employée. C'est ce qu'il fait dans Comme un silène entr'ouvert (1983-85) ou dans ses Douze tiroirs de demi-vérités pour alléger votre descente (1981-82) pour piano et orchestre. Il pourra aussi voyager rapidement d'un point à un autre de son espace harmonique par l'utilisation de pivots, comme en théorie tonale traditionnelle. Par exemple, le huitième accord de la transformation -I est identique au septième accord de la transformation -IV et cette identité permet un voyage rapide entre deux points éloignés. Finalement, il pourra faire une lecture verticale du modèle harmonique. Il enchaînera donc le premier accord de +I, -I, -II, -III, -IV, puis le deuxième accord, et ainsi de, suite. Plusieurs passages du Cactus rieur, comme d'ailleurs d'autres œuvres qui l'ont précédé (par exemple À propos... et le baron perché), sont basés sur une lecture verticale du modèle harmonique.

8 On remarquera que chaque accord peut être identifié par deux nombres séparés par un point. Par exemple, l'accord 2.3 (-II) est le troisième accord du deuxième groupe de sept en transformation -II. 


\section{Le cactus rieur}

Le cactus rieur et la demoiselle qui souffrait d' une soif insatiable a été terminé en 1986. Cette œuvre pour orchestre, fruit d'une commande de l'Orchestre symphonique de Québec, est dédiée à la chanteuse allemande Ingrid Schmithüsen. Dans les notes de programme qui accompagnent la partition, Bouliane fait allusion à un programme intime et rattache donc d'emblée son œuvre à la tradition romantique du poème symphonique. Quant aux influences possibles, il mentionne les noms de Richard Strauss et de Miles Davis.

Le cactus est une plante plutôt solitaire. Il croît, envers et contre tout et parfois doit s'hérisser d'épines... Il peut arriver qu'une jolie demoiselle égarée dans les sables aille à sa rencontre... C'est alors qu'il se découvrira des ressources aqueuses jusque là insoupçonnées et l'invitera à boire de sa chair, le cactus et la demoiselle s'enlaçant dans un sourire exquis...

$\mathrm{Ce}$ Cactus rieur... renoue peut-être avec la tradition du poème symphonique mais l'argument en serait tout de même plus secret. Il importe en définitive assez peu de suivre pas à pas une pseudo-historiette; je me suis plutôt laissé porter au fil des climats tout en contrastes suggérés par cette rencontre fantasque.

Musicalement $\mathrm{j}$ 'aime bien louvoyer aux frontières du connu et du possible... Je me suis complu ici dans un hybride imaginaire. Je me suis amusé à nouer ensembles des simili-lambeaux oubliés au fond des tiroirs de... disons Richard Strauss et Miles Davis... une rencontre bien sûr impossible... c'est tout ${ }^{9}$.

Ce texte nous en apprend somme toute assez peu. Bien que l'atmosphère générale et les inspirations possibles soient mieux définies, on n'y dit rien de vraiment concret sur la forme, si ce n'est qu'elle est une suite de « lambeaux oubliés ». Devons-nous en conclure que celle-ci n'a que peu d'importance, qu'il ne faut voir en cette œuvre au titre farfelu qu'un simple divertissement humoristique ? Le danger en est grand. Or, Bouliane est également l'auteur d'un Silène entr'ouvert, dont le titre renvoie au prologue de Gargantua de Rabelais, prologue qui contient l'avertissement suivant, qui pourrait tout aussi bien s'appliquer au Cactus rieur.

Par autant que vous, mes bons disciples, et quelques aultres foulz de sejour, lisans les joyeulx tiltres d'aulcuns livres de nostre invention, comme

9 Denys Bouliane, notes de programme pour Le cactus rieur (1986). 
Gargantua, Pantagruel, Fessepinte, La Dignité des Braguettes, Des Poys au lard cum commento, etc., jugez trop facilement ne estre au dedans traicté que mocqueries, folatreries et menteries joyeuses, veu que l'ensigne exteriore (c'est le tiltre) sans plus avant enquerir est communement receu à derision et gaudisserie. Mais par telle legiereté ne convient estimer les œuvres des humains. [...] C'est pourquoy fault ouvrir le livre et soigneusement peser ce que y est deduict. Lors congnoistrez que la drogue dedans contenue est bien d'aultre valeur que ne promettoit la boite, c'est à dire que les matieres icy traictées ne sont tant folastres comme le tiltre au dessus pretendoit ${ }^{10}$.

Il faudra donc prendre le compositeur au sérieux et, armés de la partition et d'un enregistrement ${ }^{11}$, établir nous-même la carte topographique des lieux.

\section{Survol exploratoire : la grande forme}

Une première série d'auditions permet de discerner une division en deux grandes sections suivies d'une coda. La première de ces deux sections commence et se termine sur des formules de percussion. La deuxième débute (au chiffre 57) sur une longue pédale de $f a$ en harmoniques de cordes, accompagnée d'une pédale

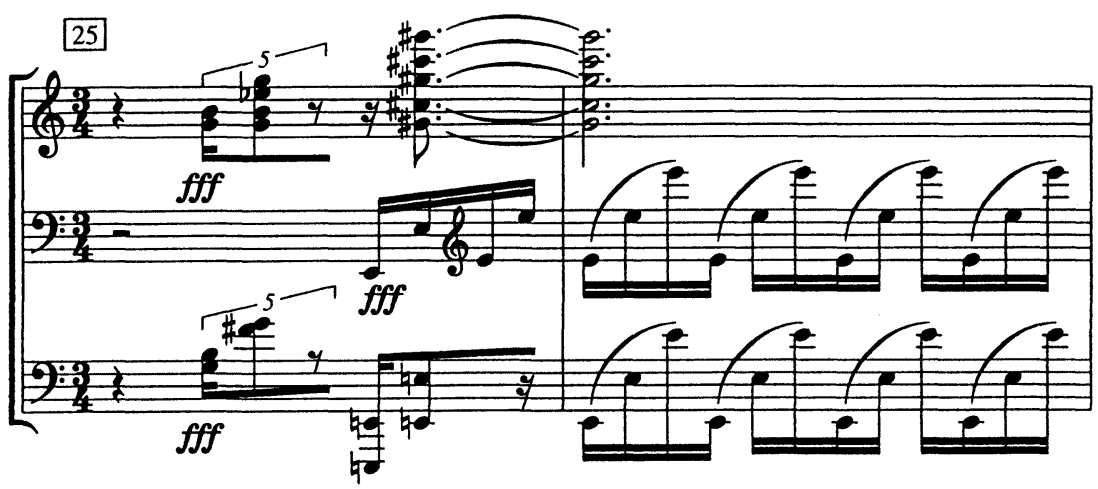

Exemple 6 : Le cactus rieur, chiffre 25.

10 François Rabelais, La vie très horrificque du grand Gargantua (1534) (Paris: GarnierFlammarion, 1968), 44.

11 La partition est disponible sur demande au Centre de musique canadienne. Un enregistrement sur disque compact, par l'Orchestre symphonique de Québec sous la direction de Simon Streatfield, est disponible sous étiquette SNE (SNE-567-CD). 
rythmique en 3/16 à la caisse claire. À l'audition, on pourra également déceler le rôle primordial accordé, dans la première section, à la note $m i$, sorte de centre vers lequel la musique revient constamment avec éclat (comme aux chiffres 25 , 35 et 54). L'exemple 6 est une réduction de l'interjection du chiffre 25 , sorte d'aboutissement d'un processus de gonflement commencé à $21^{5}$. La musique de la deuxième section, quant à elle, s'organise autour d'un nouveau centre $f a$, centre qui, nous l'avons dit, est présenté en pédale au chiffre 57.

La deuxième section semble aussi utiliser des procédés rythmiques plus développés que ceux de la première. Pour s'en convaincre, on n'a qu'à regarder de plus près la musique du xylophone, du piano, du premier violon et du premier alto à partir du chiffre 60 (exemple 7a). On retrouve ici une superposition de rythmes irréguliers qui ne semblent pas pouvoir s'organiser en couches métriques. C'estégalement dans la seconde partie que, pour la première fois, l'élément polymétrique est clairement perceptible, comme, par exemple, au chiffre 71 (exemple $7 b$ ). Ce passage superpose quatre couches métriques. Les deux voix supérieures, qui divisent chaque noire en quatre parties, font se contraster les organisations binaire et ternaire. Les trois voix inférieures utilisent le même contraste mais, cette fois, la noire est divisée en trois parties. De plus, l'émergence progressive d'un rythme de valse, à partir du même endroit, est un élément nouveau qui marque bien l'individualité de cette section de l'œuvre.

L'opposition $m i-f a$, qui est certainement le principe formel le plus facilement détectable du Cactus rieur, vient également marquer la coda, qui débute au chiffre 77. Cette dernière section est en fait une décomposition du matériel

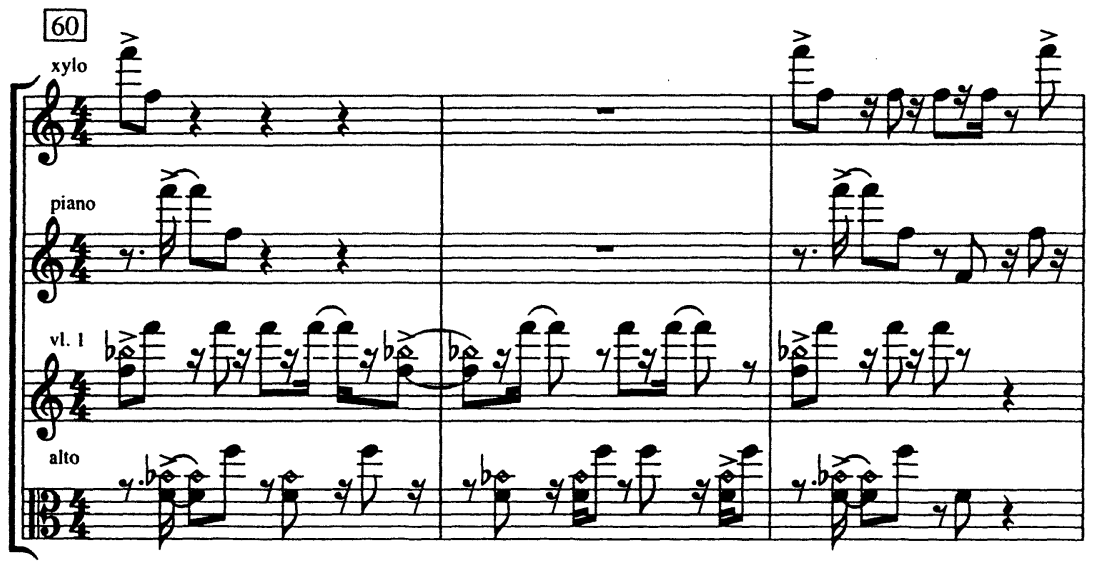

Exemple 7a : Le cactus rieur, $2^{\mathrm{e}}$ partie, chiffre 60 , mes. 1-3. 


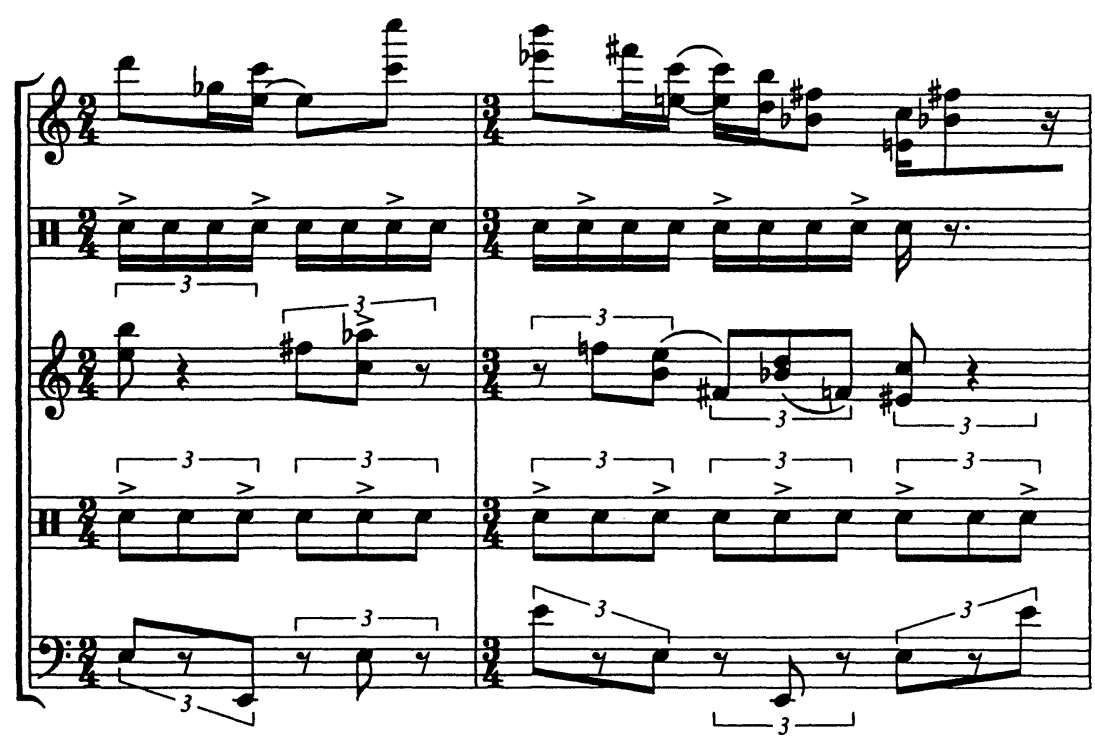

Exemple $7 \mathrm{~b}$ : Le cactus rieur, $2^{\mathrm{e}}$ partie, chiffre 71, mes. 4-5.

précédent, décomposition pendant laquelle le compositeur élimine progressivement les notes autres que $f a$ et $m i$. De 80 à 81 , il ne reste plus que ces deux notes.

Quelles sont les premières impressions générales quant à la facture de l'œuvre ? On est tout d'abord frappé par la virtuosité de l'écriture orchestrale. L'orchestre reluit, resplendit de couleurs chatoyantes, se fait tour à tour violent et tonitruant, s'immobilise dans de grandes plages brumeuses, prend un aspect d'ensemble de chambre. La musique semble passer rapidement d'un style à l'autre. Le flot musical est continuellement interrompu, souvent après seulement quelques mesures, par des silences ou des points d'orgue. On remarque les nombreuses répétitions qui donnent l'impression de revoir toujours le même objet, mais sous des angles différents. Finalement, on ne peut que se sentir désorienté face à de si fréquentes références tonales. Il ne saurait bien sûr s'agir de tonalité traditionnelle, mais il n'en demeure pas moins que le compositeur se sert de nos habitudes traditionnelles d'audition pour créer de véritables mirages tonals, qu'il prend ensuite un malin plaisir à faire disparaitre. 


\section{Raffinement du découpage}

Notre deuxième série d'auditions devrait viser un découpage plus détaillé de chacune des deux grandes sections. Les points d'arrêt et les répétitions thématiques seront ici particulièrement utiles. Plusieurs possibilités méthodologiques s'offrent maintenant à nous. La première consiste à aborder l'œuvre de façon chronologique. Cette méthode, pratique dans des analyses des œuvres de longueur restreinte, s'emploie difficilement pour des œuvres de grande envergure comme le Cactus rieur. La deuxième méthode consiste à former des ensembles de passages apparentés et à étudier chacun de ces ensembles de façon séparée. C'est ce que nous ferons ici. On verra par la suite comment ces différents ensembles sont déployés dans le temps pour donner au Cactus rieur son visage propre.

\section{Formule rythmique de transition}

Le Cactus rieur commence par la présentation d'une idée rythmique à la caisse claire, sorte de ralentissement écrit, accompagné d'un decrescendo (exemple 8). Après un silence d'environ six secondes (six noires à 58), elle est reprise en canon, puis légèrement variée. Cette idée rythmique sera utilisée à quelques reprises et aura une fonction de transition. Nous l'identifierons par la lettre $R$. La formule $R$ apparaît, nous l'avons dit, au tout début de l'œuvre. On la retrouvera ensuite à $2^{6}$ (ou sixième mesure du chiffre 2), au chiffre 7 et, finalement, à $55^{4}$. Nous avons déjà mentionné cette dernière apparition, qui sert à effectuer la transition entre les deux grandes sections de l'œuvre.

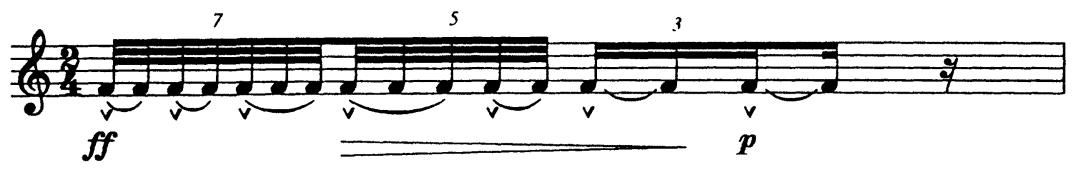

Exemple 8 : Formule rythmique de transition.

\section{Musique issue de la lecture verticale du modèle harmonique (mode desi)}

Pour pouvoir suivre cette discussion, on devra tout d'abord continuer les transformations du modèle harmonique en mode de si présentées à l'exemple 5 . La méthode d'identification des accords est celle utilisée par le compositeur. Par exemple, 2.3 (-IV) est un accord $s o l-m i-d o$, le troisième de la deuxième section de la transformation -IV à l'exemple 5. Bien entendu, chacun de ces accords est susceptible d'être coloré par différentes notes étrangères. 
Les premiers accords de l'œuvre, présentés à la première apparition des instruments mélodiques, au chiffre 1 , sont $7.3(-\mathrm{IV}), 7.4(+\mathrm{I})$ et $7.4(-1)$. Ce court passage ne dure qu'une mesure. On revient à cette musique entre les chiffres 3 et $3^{5}$. Cette fois, la citation est allongée d'un temps pour compléter l'énoncé harmonique du groupe 7.4. Le motif mélodique $m i$-ré est ensuite prolongé par une extension $f a-m i$, motif de toute première importance puisque, nous le savons déjà, il déterminera la grande forme.

On revient une troisième fois à cette musique au chiffre 7 , en se rendant cette fois jusqu'à un accord parfait de mi majeur en premier renversement (chiffre $7^{4}$ ) (exemple 9). Bouliane fait sonner cet accord de mi majeur comme un point d'arrivée logique de la progression. L'auditeur croit presque à ce semblant de pôle tonal. Cette illusion est rendue possible par le fait, déjà mentionné plus haut, que la plupart des accords de trois sons générés dans son modèle harmonique (mode de si) par les transformations -I, -II, -III et -IV sont des accords parfaits ou des accords augmentés pouvant facilement être présentés de telle façon que l'auditeur ne peut s'empêcher de les rattacher à une pseudo-tonalité.

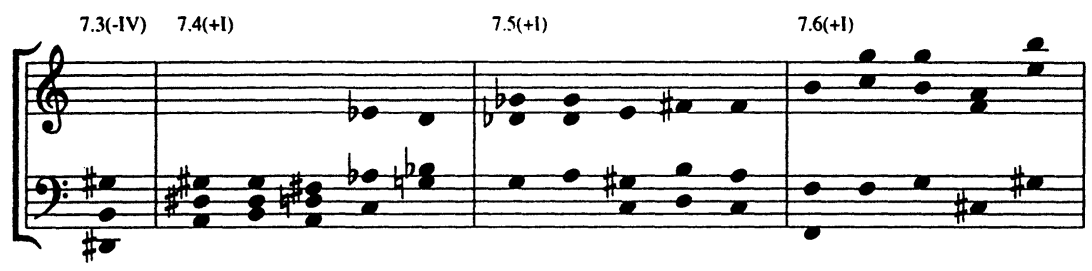

Exemple 9 : Lecture verticale, 7.3 (-IV)-7.6 (-IV).

L'accord que l'on retrouve à $7^{4}$, avec $d o$ à la basse, est aussi 1.6 (-IV). Cette ambiguiité permet au compositeur de voyager rapidement vers une autre partie de son modèle harmonique, vers des harmonies qui conviennent mieux aux grands éclats qui suivront. On trouve donc, à partir de $7^{4}$, accompagnant un arpège d'accord de septième diminuée à la basse, les accords 1.6 (-IV), 1.6 (-III), 1.6 (-II) et, à $7^{16}, 1.6$ (-I) (exemple 10). C'est maintenant mi qui semble, à l'audition, devenir un nouveau point de stabilité.

La deuxième idée mélodico-harmonique du Cactus rieur est présentée de 2 à $2^{6}$, dans l'aigu. Le matériau harmonique utilisé va de 9.3 (-I) à 9.5 (-I). Cette idée revient pendant les trois premières mesures de 10 mais est ensuite interrompue par un matériel différent. Elle revient au chiffre 11, mais cette fois couvre toute la distance de 9.3 à 10.4 (exemple 11). Le compositeur accorde une importance toute particulière aux accords parfaits majeurs de $s i$, de lab et de sib $\left(12^{3}\right.$ et $\left.12^{4}\right)$ ainsi qu'à la superposition des accords 10.3 (-IV) et 10.4 (+I). À $14^{5}$, 

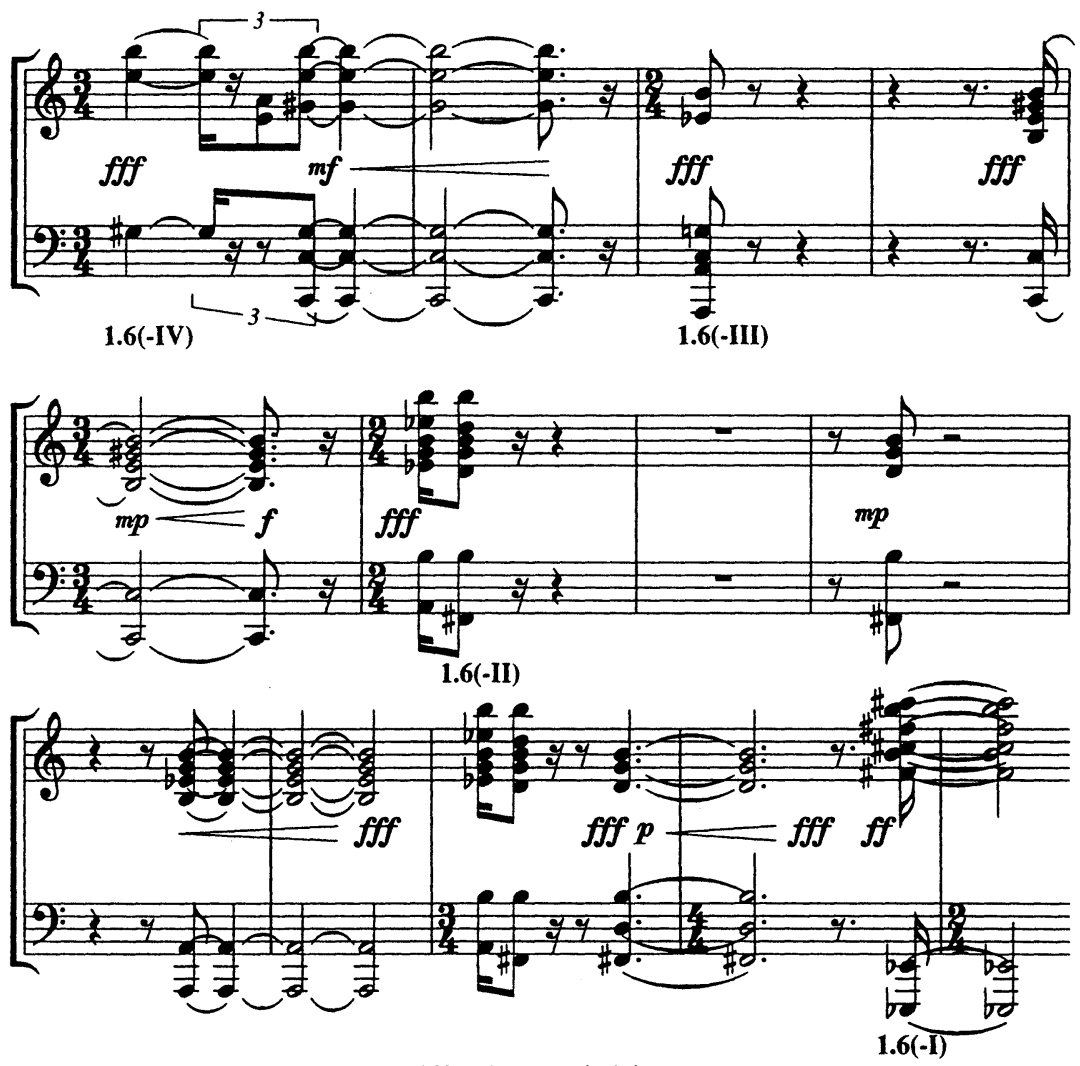

Exemple 10 : Le cactus rieur, chiffre 7, mes. 4-16.

cette combinaison d'accords commence à glisser progressivement, ce qui nous amène, quatre mesures plus tard, à une nouvelle division formelle.

Le troisième emprunt à la lecture verticale commence au chiffre 18 et se termine au deuxième temps de $21^{5}$ dans une succession de courts segments de caractères différents. Il s'agit d'une réalisation de 10.4 (-IV) à 11.7 (+I). Au quatrième temps de $21^{5}$, le compositeur, qui veut s'acheminer vers un point culminant, décide d'enchaîner directement avec les sections 1.7 (+I) à 2.2 (-IV) de la lecture verticale composée, qui est sans doute le passage qui se prête le mieux à cette utilisation. Ce déplacement se fait sans heurt, puisque 11.7 (-I) et $1.7(+\mathrm{I})$ ont deux notes communes et une troisième à distance d'un ton. Cette progression nous mène tout d'abord vers ré majeur, puis vers un accord pentacordal de type 5-35 [02479], réalisé comme un accord de septième de domi- 

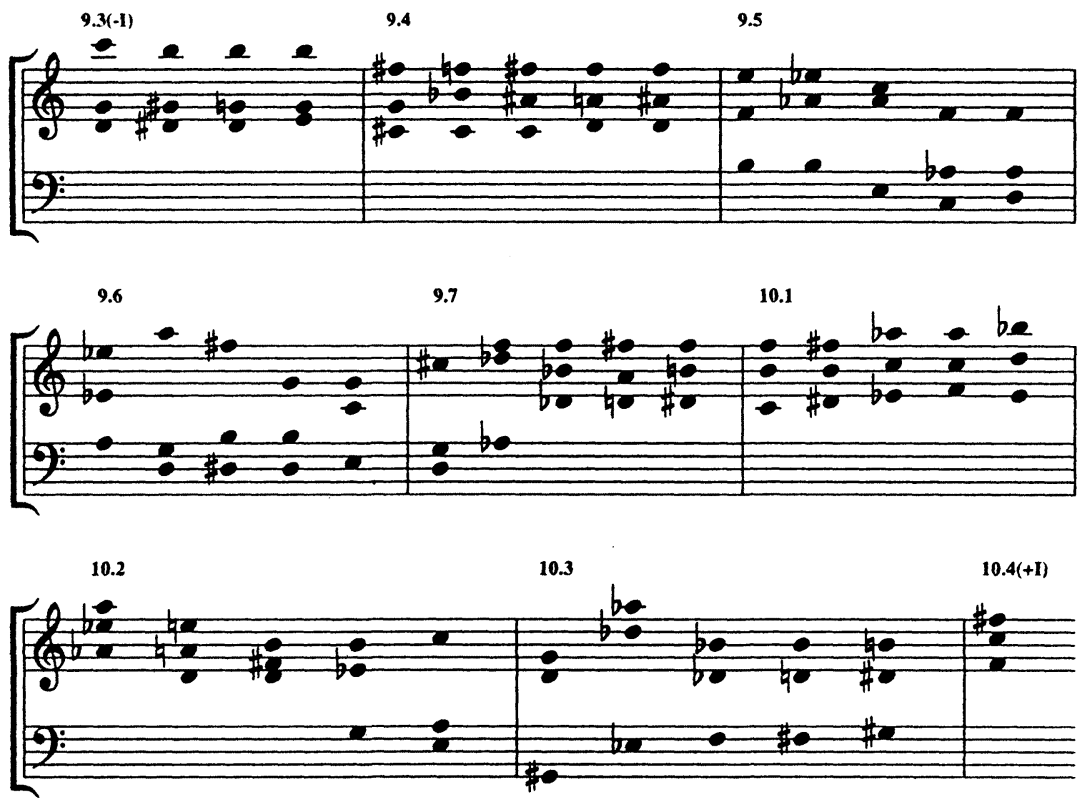

Exemple 11 : Lecture verticale, $9.3(-\mathrm{I})-10.4(+\mathrm{I})$.

nante sur $d o \sharp$ avec un $f a \#$ ajouté. L’accord final, $d o \sharp$ mineur en premier renversement (et non $f a \#$, comme prévu), arrive au chiffre 25 et laisse finalement toute la place au $m i$ de la basse jusqu'au chiffre 26 .

Comme partout ailleurs dans cette première partie du Cactus rieur, Bouliane passe rapidement d'une atmosphère à l'autre, alternant les styles. Il semble que, dans son esprit, certains accords ou progressions soient rattachés à un type précis de gestuelle orchestrale ou à un style de musique particulier. Dans le passage qui nous occupe, par exemple, le caractère conjoint de la réalisation à trois voix l'entraîne vers un romantisme quasi-mahlérien.

On remarquera également le canon entre trombones et trompettes, rythmiquement inexact, de la deuxième à la quatrième mesure de 21 (exemple 12). Ce type de canon est un des moyens utilisés régulièrement par Bouliane afin de créer l'impression d'ambiguïté métrique qu'il recherche. Dans ce passage, puisqu'il était déjà à peu près impossible de percevoir un mètre stable à cause du caractère hachuré de la musique, le canon est utilisé comme étape transitoire entre la musique pratiquement amétrique de 18 à 21 et celle, plus régulière, de 23 . 

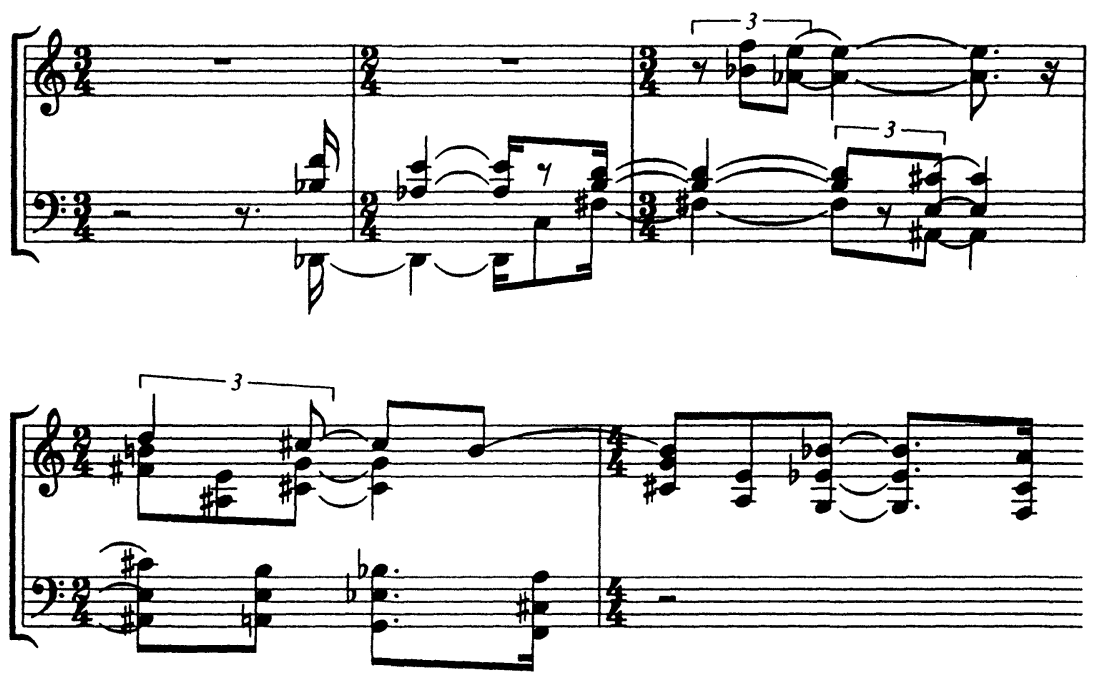

Exemple 12: Le cactus rieur, chiffre 21, mes. 1-5 (trompettes et trombones).

Au chiffre 36, la musique se transforme brusquement et adopte un style vaguement inspiré de celui du jazz moderne, en plus complexe (il s'agit peut-être de la référence à Miles Davis mentionnée par Bouliane dans ses notes de programme). De 36 à 43, Bouliane tire l'essentiel de son matériau mélodicoharmonique des sections 2.3 à 4.4 (-II) de la lecture verticale. On remarquera l'emploi systématique de mixtures (fausses résonances dissonantes), parfois présentées en canons irréguliers, et la superposition de formules mélodiques rapides aux instruments graves. Ces formules sont construites peu à peu, atteignent leur sommet entre $39^{5}$ et 40 , puis sont décomposées. À partir de $41^{4}$, la flûte, la première clarinette et les trombones se détachent de l'ensemble dans ce qui pourrait être qualifié d'accompagnement libre.

Après une courte parenthèse, on revient à la lecture verticale de 44 à 47 . Cette fois, on couvre la distance de 4.4 (-III) à 5.7 (+I), encore une fois avec mixtures, formules mélodiques aux instruments graves et accompagnement libre. Suit un long point d'orgue (environ 11 secondes), réunissant les accords 3.7 (-II) et 4.1 (-II). Après ce point d'orgue, on enchaîne avec la section 12.1 (-III) de la lecture verticale. Le piano est traité en soliste et rappelle les Douze tiroirs de demivérités. Au chiffre 49 , à cause du caractère circulaire du modèle harmonique, nous nous retrouvons au début de la lecture verticale (exemple 13). Le matériau de base est ici fortement développé, surtout sous forme d'arpèges et d'éclats bruyants. 


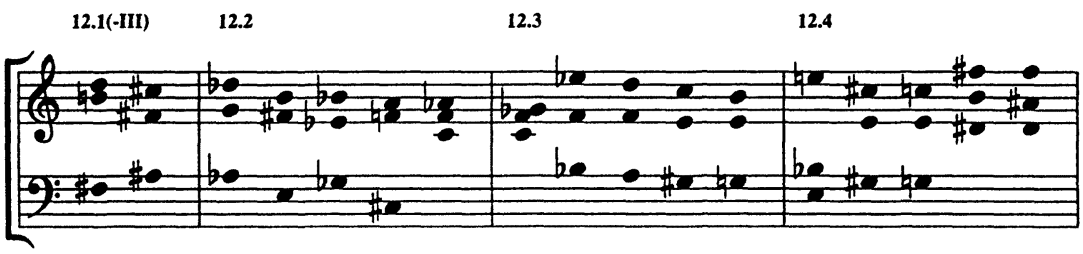

12.5

12.6

12.7

1.1

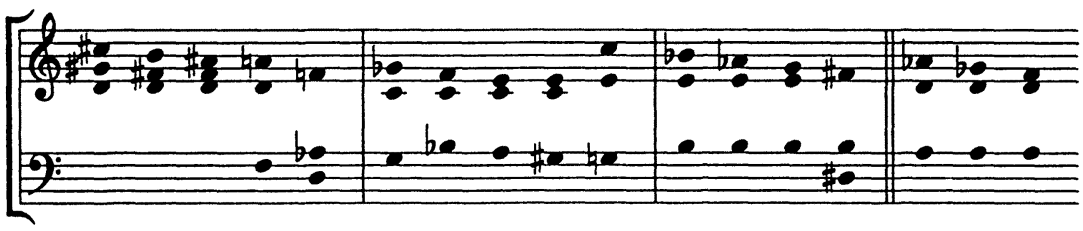

Exemple 13 : Lecture verticale, 12.1 (-III)-1.1 (-III).

Trois mesures avant le chiffre 50, le compositeur décide de ramener son point culminant du chiffre 25 . Il commence donc à introduire, en arpèges ascendants, l'accord $1.7(+\mathrm{I})$, qui lui permettra de revenir au matériel présenté plus tôt. La citation est exacte (à l'exception d'une réorchestration presque imperceptible), de $50^{2}$ à 55 . On trouve donc une deuxième confirmation de la primauté de mi. Cet épisode vient clore de façon grandiose la première partie de l'œuvre.

La dernière citation de la lecture verticale se trouve au début de la deuxième partie. Elle possède un caractère très différent de celui des précédentes et sera traitée plus loin, dans une section consacrée spécifiquement à la deuxième partie du Cactus rieur.

\section{Emprunts et citations}

Les personnes qui entendent le Cactus rieur pour la première fois et qui connaissent bien la production de Denys Bouliane noteront certainement que de nombreux passages de l'œuvre pour orchestre ont été empruntés directement à deux autres de ses œuvres : À propos... et le baron perché ? $(1985)^{12}$ et $A$

12 À propos... et le baron perché ?, composé en 1985, est le résultat d'une commande de la Société de musique contemporaine du Québec. Écrite pour deux clarinettes basses, deux contrebassons, deux cors, une trompette, deux trombones et une contrebasse, l'œuvre a été créée le 13 mars 1986 à Montréal. Il en existe un enregistrement par l'Ensemble de la SMCQ sous la direction de Walter Boudreau (Société nouvelle d'enregistrement SNE-CD-543). On peut obtenir la partition au Centre de musique canadienne. 
Certain Chinese Cyclopadia $(1986)^{13}$. Comme un silène entr'ouvert (1983$85)^{14}$ a aussi exercé une influence certaine sur le Cactus, puisque le système harmonique des deux œuvres est identique. On peut identifier les citations de façon chronologique.

Le premier emprunt apparaît aux mesures $3^{6}$ et $3^{7}$, et prend la forme d'une descente nous menant de 7.4 (-IV) à un accord de ré majeur-mineur, qui servira de point de repos pseudo-cadentiel. Cette descente vient de laChinese Cyclopadia, du deuxième temps de $19^{3}$ à la fin de $20^{1}$, qui contient l'accord de ré. Au chiffre 4 du Cactus, l'accord est prolongé en écho avec mixture de quinte à la flûte. La deuxième citation commence au chiffre 5 et se termine à la fin de $6^{1}$. On retrouve ce passage à la fois dans le Baron perché (chiffre 6, mais seulement les dix premières noires) et dans la Chinese Cyclopadia (chiffre 13). Cette courte citation est très importante pour le Cactus puisque tout un passage sera plus tard construit à partir de son motif principal, basé sur l'alternance 1.1 (-IV) et 1.2 (-IV) (exemple 14). À $6^{2}$, on passe à une autre citation de la Chinese Cyclopadia (de $58^{4}$ à 59). La transition se fait sans problème puisque le dernier accord de la citation précédente et le premier de celle-ci ont trois notes communes et un mouvement chromatique sol-solb.

5

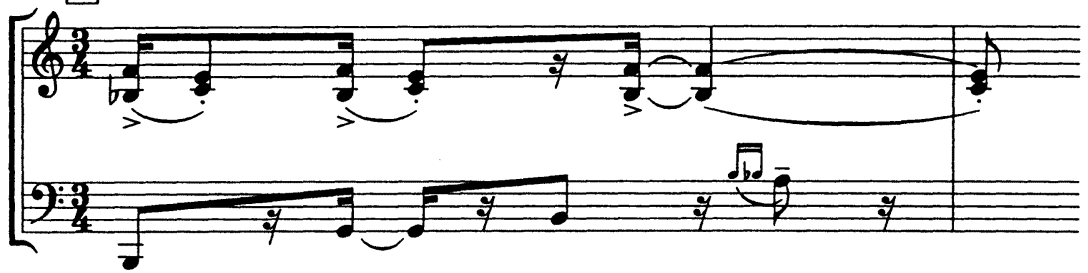

Exemple 14 : Le cactus rieur, chiffre 5 .

13 A Certain Chinese Cyclopadia, composé en 1986, est le résultat d'une commande de l'ensemble York Winds de Toronto. Écrite pour flûte, cor anglais, clarinette, cor et basson, l'œuvre a été créée le 22 juillet 1988 par le quintette à vent Albert Schweitzer. Il en existe un enregistrement par le quintette Calamus (Société nouvelle d'enregistrement SNE-567-CD). On peut obtenir la partition au Centre de musique canadienne.

14 Comme un silène entr'ouvert, composé entre 1983 et 1985, est le résultat d'une commande de l'Ensemble Köln. Écrite pour petite flûte, hautbois, clarinette basse, trombone, harpe, piano et contrebasse, l'œuvre a été créée dans sa version finale le 9 mai 1986 par l'Ensemble Köln sous la direction de Robert HP Platz. Il en existe un enregistrement par les mêmes interprètes (Société nouvelle d'enregistrement SNE-CD-543). On peut obtenir la partition au Centre de musique canadienne. 
Au chiffre 8 commence une section d'un romantisme exacerbé, dans un langage vaguement influencé par Strauss et Berg, à la postmoderne. Elle se terminerad'une façon magnifique sur une simple tierce mib-sol (juste avant 10). On ne peut éviter d'entendre ce mib comme un centre tonal, surtout après un passage contenant autant de références aux gestes caractéristiques du passé. Cette musique est empruntée au Baron perché (de 59 à 62). Les valeurs sont doublées, probablement pour faciliter la mise en place. La quatrième mesure du chiffre 10 cite une nouvelle fois la descente qui, plus tôt, avait mené à l'accord de ré majeur-mineur.

Nous arrivons maintenant à une section du Cactus faisant de nombreuses références à la Chinese Cyclopadia. La mesure $14^{9}$ cite, à la fin du grand glissement mentionné plus haut, une mesure du quintette à vent $\left(13^{4}\right)$, suivie de deux mesures d'extension. Au chiffre 15, on retrouve une descente rapide tirée des deux premières mesures du chiffre 4 du quintette. À partir du dernier temps de la deuxième mesure de 15 , le compositeur continue avec les cycles de quintes typiques de la Cyclopaedia, sans qu'il s'agisse (du moins à ce qu'il semble) d'une véritable citation. La musique se décompose progressivement et, au chiffre 16 , on recommence à citer le quintette de façon littérale. La première mesure du chiffre 16 du Cactus correspond à $16^{3}$ de la Chinese Cyclopadia. Cet épisode se termine à la dernière mesure de 16.

On enchaîne immédiatement, à 17, avec une autre citation du quintette (de 6 à 7). La musique tourne autour de la tierce $f a \sharp-l a \sharp$ (noté $s i b$ ), pour finalement aboutir à un accord parfait de la mineur en deuxième renversement. Ce dernier passage, amputé de la dernière mesure, est répété plus loin, au chiffre 43.

Il est possible de discerner une certaine logique dans cet enchaînement particulièrement hétéroclite de citations de la Chinese Cyclopadia. La première (à $14^{9}$ ) commence par une succession do -la-ré à la basse, superposée au motif de l'exemple 13, avec do comme note supérieure. La descente en cycle de quintes permet une décomposition progressive pour arriver, au chiffre 16, à une musique raréfiée. Bouliane se sert alors d'un passage du quintette possédant ce caractère en son début et montrant par la suite une reprise de l'activité, aboutissant, à la mesure avant le chiffre 17, à l' accord sur lequel la section avait commencé.

Pour un aperçu complet des citations, le lecteur pourra consulter le tableau d'ensemble de la forme donné plus loin (tableau 3).

\section{Insertion libre}

Entre les chiffres 26 et 36, Bouliane compose un des plus extraordinaires passages de son œuvre. Il s'agit d'une grande section libre, très heurtée, riche en 
Tableau 3 : Tableau d'ensemble de la forme

\section{Première partie}

\begin{tabular}{|c|c|c|c|c|}
\hline$\#$ & LECTURE VERTICALE & $R$ & EMPRUNTS & DIVERS \\
\hline 0 & & $\mathbf{R}$ & & \\
\hline 1 & $7.3(-$ IV $)$ à $7.4(-$ II $)$ & & Baron perché (57) & \\
\hline 2 & $9.3(-\mathrm{I})$ à $9.5(-\mathrm{I})$ & & & \\
\hline $2^{6}$ & & $\mathbf{R}$ & & \\
\hline 3 & $7.3(-$ IV $)$ d $7.4(-$ IV $)$ & & Baron perché (57) & extension $f a-m i$ \\
\hline $3^{6}$ & & & Chinese cyclopedia (193-201) & vers ré (écho au ch. 4) \\
\hline 5 & & & $\begin{array}{l}\text { Chinese cyclopedia (13) } \\
\text { Baron perché (6) }\end{array}$ & 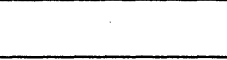 \\
\hline $6^{2}$ & & & Chinese cyclopedia (584-59) & \\
\hline 7 & & $\mathbf{R}$ & & \\
\hline $7^{1}$ & 7.3 (-IV) а 7.4 (-IV) & & Baron perché (57-577) & \\
\hline $7^{4}$ & $1.6(-I V a ̀-I)$ & & & vers mi bémol \\
\hline 8 & & & Baron perché (59-62) & vers mi bémol \\
\hline 10 & $9.3(-1)$ aे $9.5(-1)$ & & & \\
\hline $10^{4}$ & & & Chinese cyclopedia (193-201) & \\
\hline 11 & $9.3(-\mathrm{I}) \mathrm{a} 10.4(+\mathrm{I})$ & & & glissement (145) \\
\hline $14^{9}$ & & & Chinese cyclopedia (134) & extension de 2 mesures \\
\hline 15 & & & Chinese cyclopedia (41-42) & \\
\hline $15^{2}$ & & & & cycles de quintes \\
\hline 16 & & & Chinese cyclopedia (163-17) & \\
\hline 17 & & & Chinese cyclopedia (6-7) & vers $l a(170)$ \\
\hline 18 & $10.4(-\mathrm{IV})$ a $11.7(+\mathrm{I})$ & & & \\
\hline $21^{5}$ & $1.7(+1)$ a $2.2(-I V)$ & & Baron perché (13-21) & vers $m i(25)$ \\
\hline 26 & & & & section libre (-IV) \\
\hline 32 & & & & à la Stravinsky, vers $m i$ \\
\hline 36 & $2.3(+\mathrm{I}) \mathrm{a} 4.4(-\mathrm{II})$ & & Baron perché (21-33) & formules mélodiques \\
\hline $41^{4}$ & & & & accompagnement libre \\
\hline 43 & & & Chinese cyclopedia (6-7) & \\
\hline 44 & $4.4(-$ III $)$ a $5.7(+1)$ & & Baron perché (33-38) & \\
\hline $46^{I 1}$ & & & & point d'orgue \\
\hline 47 & $12.1(-\mathrm{III}) \mathrm{a} 1.3(-\mathrm{IV})$ & & Baron perché (1-4) & vers $m i(493)$ et $(42-44)$ \\
\hline $50^{2}$ & $1.7(+\mathrm{I}) \mathrm{a} 2.2(-\mathrm{IV})$ & & Baron perché (13-21) & vers $m i(54)$ \\
\hline $55^{4}$ & & $\mathbf{R}$ & & \\
\hline
\end{tabular}

\section{Deuxième partie}

\begin{tabular}{|l|l|l|}
\hline \multicolumn{1}{|c|}{ ÉVÉNEMENT } & PROGRESSION HARMONIQUE \\
\hline 57 & $\begin{array}{l}\text { pédale de } f a \text { (cordes, puis piccolo, flûte, xylophone et piano) } \\
+ \text { pédale rythmique (3/16) (caisse claire) }\end{array}$ & \\
\hline 60 & + mélodie en harmoniques (violon II) & \\
\hline $60^{7}$ & + formule de six notes en cycle de 12 noires & \\
\hline 62 & - mélodie en harmoniques (violon II) & verticale de $6.3(-1)$ à $6.6(+I)$, jusqu'à $68^{4}$ \\
\hline 63 & + mélodie en $3 / 16$ & \\
\hline 67 & + fa dièse dans la pédale de $f a$ & \\
\hline $68^{4}$ & + rythme de valse (triolets de noires) & \\
\hline $70^{3}$ & + pédale de mi & \\
\hline 71 & & horizontale de $6.5(-1)$ à $5.1(-I)$, jusqu'à $77^{3}$ \\
\hline 72 & + motif & \\
\hline 77 & Coda & \\
\hline
\end{tabular}


contrastes. Elle commence par le motif de l'exemple 14 qui, rappelons-le, est basé sur une alternance de 1.1 (-IV) et 1.2 (-IV). On retrouve d'autres motifs importants : une septième majeure ré-mib, un arpège rapide descendant et une gamme par tons entiers en tierces majeures. Ces motifs sont sans cesse répétés, ce qui crée l'impression de toujours repartir à neuf après de brusques arrêts. En fait, n'est-ce-pas là l'impression laissée par toute la première partie du Cactus rieur? On tourne en rond et les points d'arrivée se révèlent n'être que des mirages. L'harmonie de cette section libre semble être basée sur une lecture horizontale de la transformation -IV du modèle harmonique. Il s'agit des accords qui sont à la base du Silène entr'ouvert, mais dans une réalisation on ne peut plus différente.

Au chiffre 32, le motif principal devient un ostinato. Ce passage fait penser à Stravinski, impression confirmée par l'utilisation caractéristique de la grosse caisse à $32^{6}$ et $32^{7}$, qui rappelle l'introduction à la « Glorification de l'élue » du Sacre du printemps. Bouliane continue de développer cette idée jusqu'à ce qu'il arrive, à 35, à l'accord 3.7 (-IV) (si majeur avec $m i$ à la basse). Encore une fois, il efface toutes les notes sauf miet crée par le fait même un autre point culminant, analogue à ceux de 25 et de 54 .

\section{Analyse de la deuxième partie}

L'auditeur, même inattentif, ne peut manquer de remarquer la grande différence qui existe entre la musique qui commence aux chiffres 57 et 58 et celle qui l'a précédée. Nous avons déjà mentionné quels en sont les éléments les plus marquants : pédale de $f a$, pédale rythmique, polymétrie. Voyons maintenant comment ces éléments sont combinés par le compositeur.

La pédale de $f a$ sera présente jusqu'à $78^{2}$, c'est-à-dire presque jusqu'à la fin. Tout au cours de cette grande section formelle, elle sera confiée à différents instruments et prendra de multiples visages. À partir de 57, elle est confiée aux cordes en harmoniques. Bouliane varie les sonorités en utilisant des harmoniques artificielles à la quarte juste ou à la tierce majeure et en faisant jouer sur différentes cordes sul ponticello. Le xylophone et le piano entrent à 59, suivis de la flûte et du piccolo. Il en résulte un canon rythmique irrégulier à deux voix entre ces quatre instruments, auxquels se joignent le premier violon et le premier alto (voir exemple 7a). La pédale rythmique en $3 / 16$ est confiée à la caisse claire à partir de 57 et reste présente jusqu'à 74 . À 60 , les deuxièmes violons commencent la présentation d'une mélodie en harmoniques avec mixture de quinte. Cette mélodie se continuera jusqu'à 62 .

Le prochain élément à venir se superposer à tous ceux énumérés jusqu'ici fait son apparition, de façon encore embryonnaire, à $60^{7}$. Ce n'est qu'à partir de $61^{8}$ 
qu'il aura atteint sa forme définitive. Il s'agit d'une formule de six notes, avec mixture de quinte, présentée dans un rythme en $2 / 4$ avec cinq attaques par mesures. Il faut donc six mesures (12 noires) pour revenir au début du cycle (exemple 15).

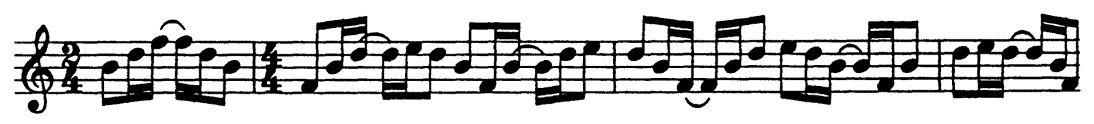

Exemple 15 : Le cactus rieur, $2^{\mathrm{e}}$ partie (formule de six notes), chiffre 61, mes. 8.

Au chiffre 63 (cor anglais et hautbois), Bouliane attache un élément mélodique à la pédale rythmique en $3 / 16$. Ce nouvel élément mélodique prendra de plus en plus d'importance et, à partir de $64^{6}$, sera combiné avec la formule de l'exemple 15.

La pédale de $f a$ est enrichie de certaines incursions du $f a$ à partir du chiffre 67. Le résultat général est donc de plus en plus touffu. La prochaine étape du développement est particulièrement cruciale. Une nouvelle idée vient encore s'ajouter à toutes ces couches à $68^{4}$ (caisse claire). Cette fois, nous avons affaire à un rythme à trois temps pour chaque mesure notée en $2 / 4$. Ce rythme se transforme rapidement en rythme de valse, surtout après l'entrée d'un ostinato de $m i$ à la contrebasse $\left(70^{3}\right)$.

Arrêtons-nous au chiffre 72 et examinons la situation générale. Nous notons maintenant une superposition des éléments suivants: pédale aiguë de $f a$ enrichie du $f a$, pédale rythmique en $3 / 16$, alternance de formules mélodiques et mélodie de valse à trois temps sur pédale de $m i$ aux contrebasses (en triolets de noires).

Qu'en est-il de l'harmonie ? En plus des sonorités créées par les superpositions, on retrouve, particulièrement à partir de 62 , deux grandes progressions. $\mathrm{La}$ première est une lecture verticale du modèle harmonique de $6.3(-\mathrm{I})$ à $6.6(+\mathrm{I})$. Ce dernier accord est joué par les premiers violons à $68^{4}$. Cette progression est bien camouflée et demande, pour son déchiffrage, une lecture attentive de la partition. La deuxième progression harmonique contrôle la valse, dont le matériel est réparti entre les vents et les deuxièmes violons. Cette fois, nous avons affaire à une lecture horizontale du modèle harmonique en transformation -I, à partir de l'accord 6.5 (exemple 16).

La mesure qui précède le chiffre 75 contient des points d'orgue sur les deuxième et troisième temps. Bouliane met ces deux temps en évidence pour deux raisons : tout d'abord pour une raison stylistique, puisque ce genre de ralentissement est typique de la valse ; ensuite pour une raison structurelle, car nous arrivons à la fin d'un cycle harmonique et au début d'un autre, avec 12.7 
71
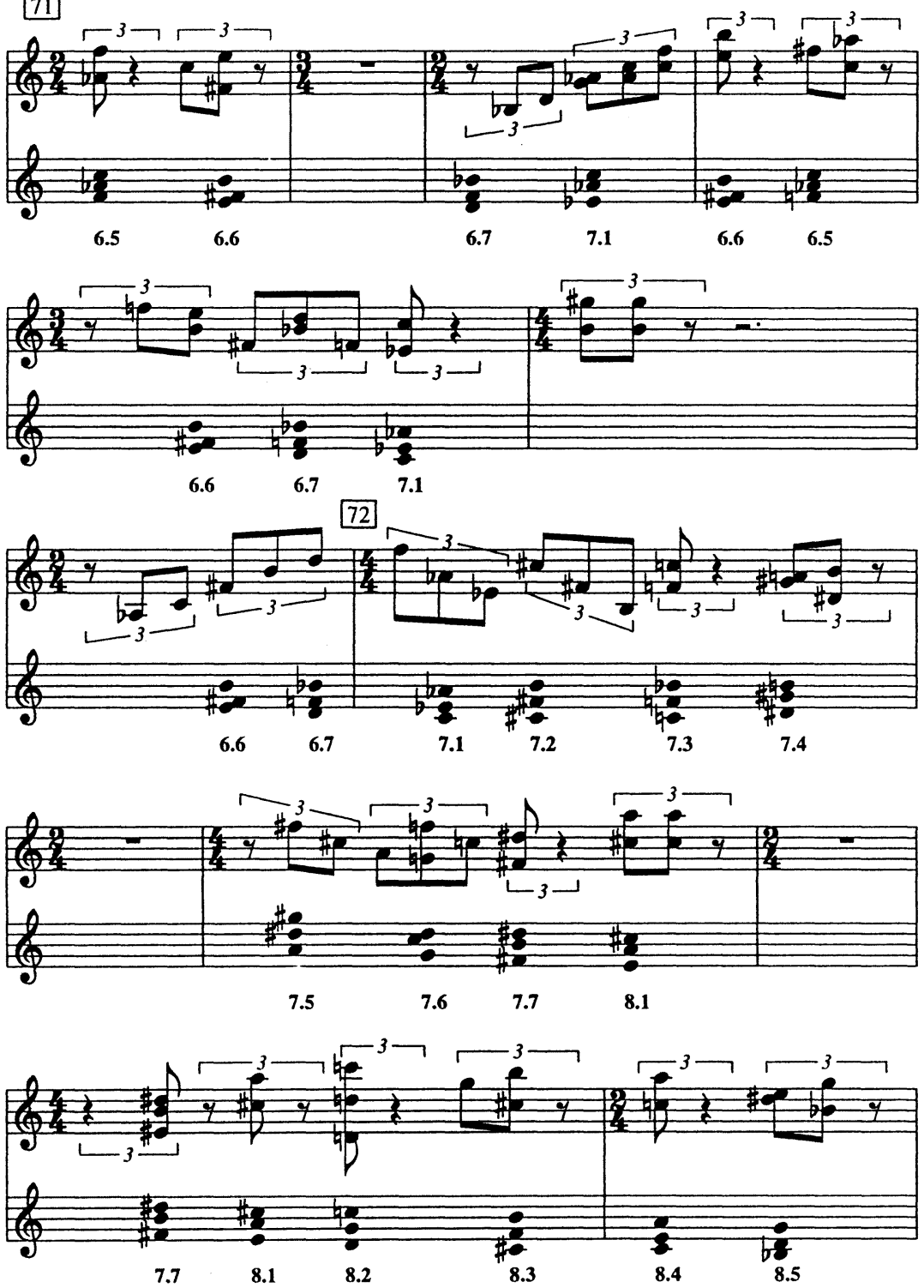

Exemple 16 : Le cactus rieur, chiffre 71, deuxièmes violons (transformation -I en lecture horizontale). 
(-I) et $1.1(-\mathrm{I})$. La valse se termine à $77^{3}$. Le reste n'est qu'une décomposition progressive vers le motif $f a-m i$.

\section{Le labyrinthe}

Écoutons encore une fois le Cactus rieur. Pour un moment, nous croyons avancer, puis nous revenons sur nos pas. Soudain, nous croyons reconnaitre une musique déjà entendue, mais cette impression se révèle trompeuse. Parfois, nous pensons être enfin arrivé au but, nous croyons avoir bien en main une tonique affirmée, mais celle-ci s'évapore soudainement et la musique replonge dans une autre direction. Notre mémoire est continuellement chatouillée par de nombreuses allusions stylistiques, par plusieurs citations, comme autant de portes s'ouvrant sur de mystérieuses chambres secrètes. Nous sommes bel et bien dans un labyrinthe, un labyrinthe aux éclairages changeants, aux harmonies elles-même labyrinthiques, basées sur des transformations modales labyrinthiques elles aussi.

Alors que la fin de la première partie du Cactus marque un retour au point de départ, à la formule rythmique du début, la musique s'oriente tout à coup dans une direction opposée et nous fait découvrir un paysage bien différent. Dans cette portion du labyrinthe, les sons de chacun des couloirs s'additionnent pour donner finalement un paysage sonore général. Sur de petites buttes, on arrive même à deviner, au loin, certaines des pièces étranges de notre première excursion.

Ce labyrinthe a-t-il une sortie ? Certainement pas dans le Cactus rieur, car le paysage semblera se figer bien avant que nous ayons pu atteindre le véritable but. Qu'aurions-nous pu trouver à la sortie ? Et cette sortie, existe-t-elle vraiment ? N'est-elle pas, elle aussi, rien d'autre qu'un mirage?

\section{Résumé}

L'abandon, il y a maintenant environ 25 ans, des techniques sérielles d'organisation du matériau musical a rendu très difficile, pour les jeunes compositeurs, le choix du type de langage à employer. Cette absence de lingua franca a poussé les musiciens à explorer quantité de façons nouvelles d'organiser leur musique. Plusieurs d'entre elles n'ont pas eu la vie longue, d'autres se sont avérées plus prometteuses. Nous nous penchons ici sur une solution intéressante au problème du contrôle harmonique proposée par un compositeur québécois, Denys Bouliane, et sur son utilisation dans une œuvre importante pour orchestre, Le cactus rieur et la demoiselle qui souffrait d' une soif insatiable. 